\title{
4 - SMARCA4 REDIRECTS BINDING OF MACROPHAGE ACTIVATING TRANSCRIPTION FACTOR 1 (ATF1) FROM GENES FOR INFLAMMATION RESOLUTION TO GENES FOR ERYTHROCYTE RESOLUTION
}

\author{
A Seneviratne 1, L Cave 1, A Shaikh 1, D Carling 2, JC Mason 1, DO Haskard 1, JJ Boyle 1.
} 1 - National Heart and Lung Institute, 2 - London Institute of Medical Sciences,

Imperial College London

Atherosclerosis is inflammatory. The resolution of inflammation, including atherosclerotic inflammation, is an emerging area. Genome wide association studies (GWAS) have revealed a number of risk loci, some of which are associated with the location of SMARCA4, a SWI/SNF chromatin remodelling gene important for gene activation. We tested whether SMARCA4 is an independent atherosclerotic risk gene with a patho-mechanism relevant to atherosclerosis. We find that SMARCA4 and LDLR are genetically independent in large scale genetic association studies. We describe an AMPK/MSK1/H3S10p pathway that modifies chromatin in response to heme. This pathway results in gene-selective H3S10 phosphorylation, seen with two classically cyclic-AMP-responsive genes, FOS and NR4A2, but not the heme response gene HMOX1. At the HMOX1 cis-regulatory sequence, chromatin looping and recruitment of SMARCA4 occurred prior to recruitment of p-ATF1. Knockdown of SMARCA4 suppressed p-ATF1 binding to HMOX1 but increased its binding to the cyclic-AMP response elements of the enhancers for FOS and NR4A2. This resulted in suppressed HMOX1 mRNA levels, but increased mRNA levels for FOS and NR4A2. Downstream, si-SMARCA4 allowed heme to induce PLA2G7, which encodes platelet-activating-factor acetyl hydrolase (PAF-AH). These data point to a role for SMARCA4 in chromatin remodelling in advance of ATF1, with the ability to alter its preferred target genes. Taken together, these data indicate that SMARCA4 is an independent atherosclerosis risk gene, is associated with a novel mechanism, and is involved in switching between leukocyte-resolution and erythrocyte-resolution.

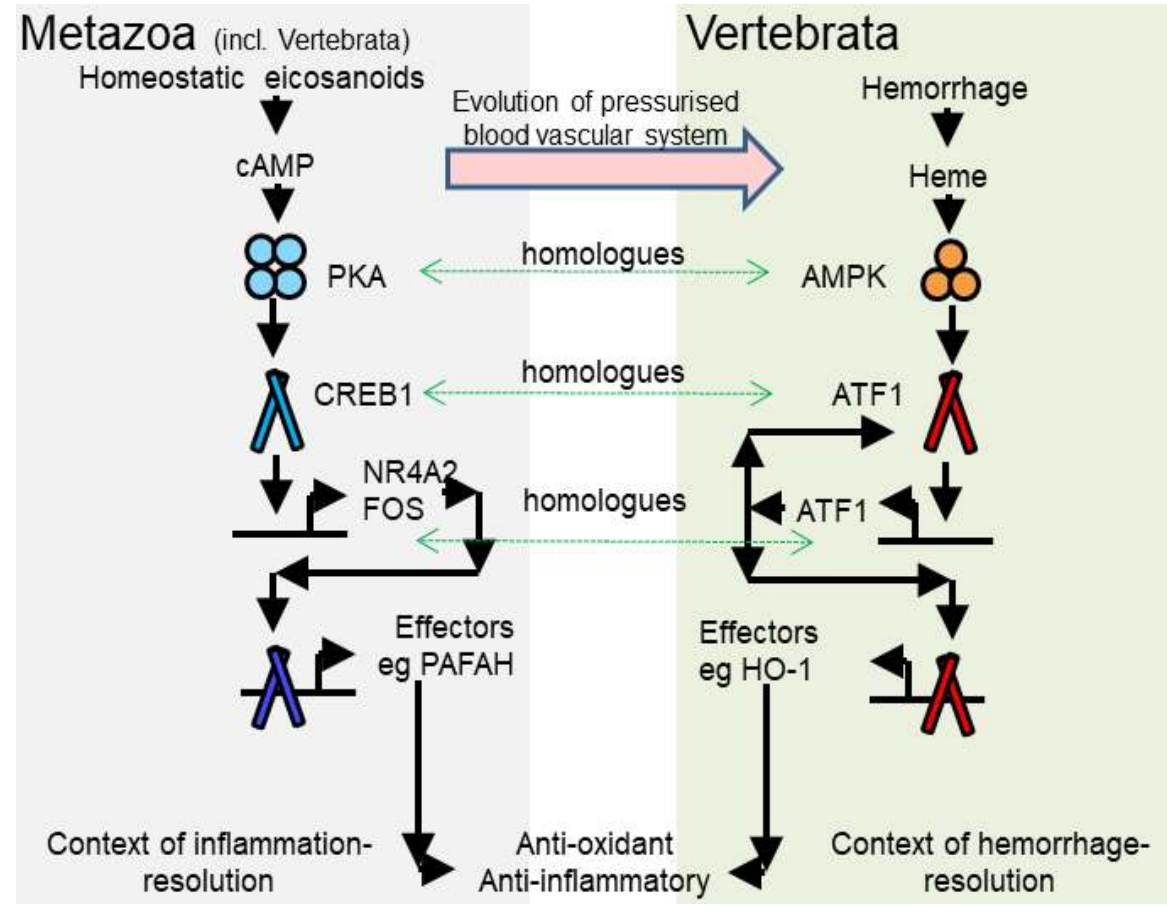

Figure 1 A, Putative evolution of ATF1-mediated hematoma resolution relative to CREB1 mediated inflammation-resolution Left grey box - older mechanism found in both activate macrophagest to elevete intraceedlllarar c-AMP. This leads to a classical pathway involving
CREB1-phosphorylation, 'homing' to c-AMP response elements and inducing target genes. CREB1-phosphorylation, 'homing' to C-AMP response elements and inducing target genes
Initially, these are FOS and NR4A2, but in turn these regulate genes that suppress inflammation such as PLA2G7 (PAFAH). Right pale green box -AMPK is a homologue of PKA and is activated by heme. AMPK activates ATF1. This activates an alternative set of genes that also second pathway evolved in response to tissue hemorrhages, which are likelier with the
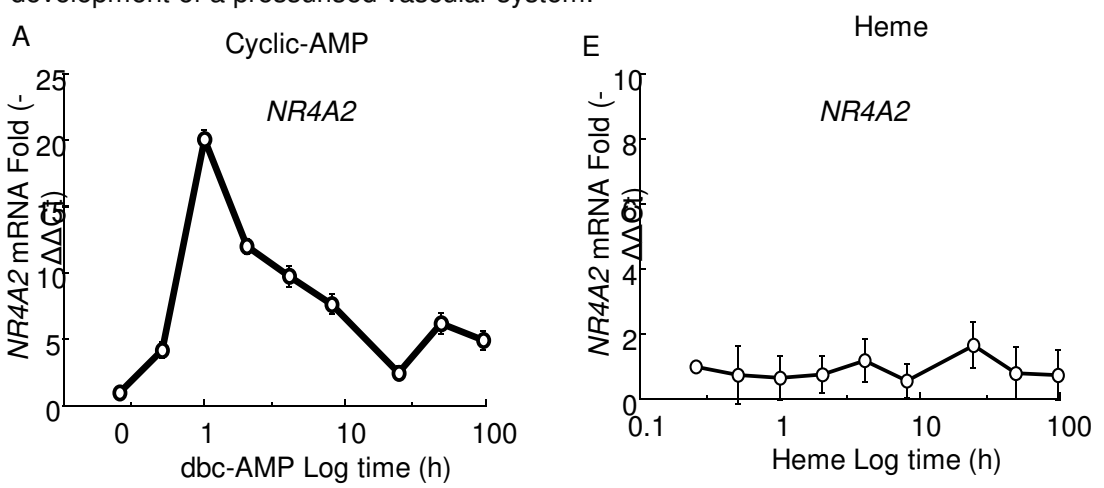

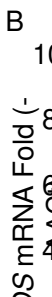

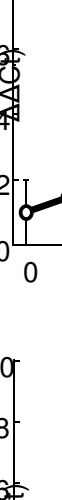
ros

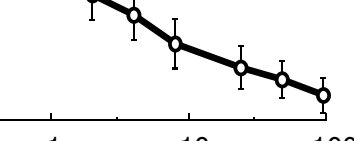
abc-AMP Log time
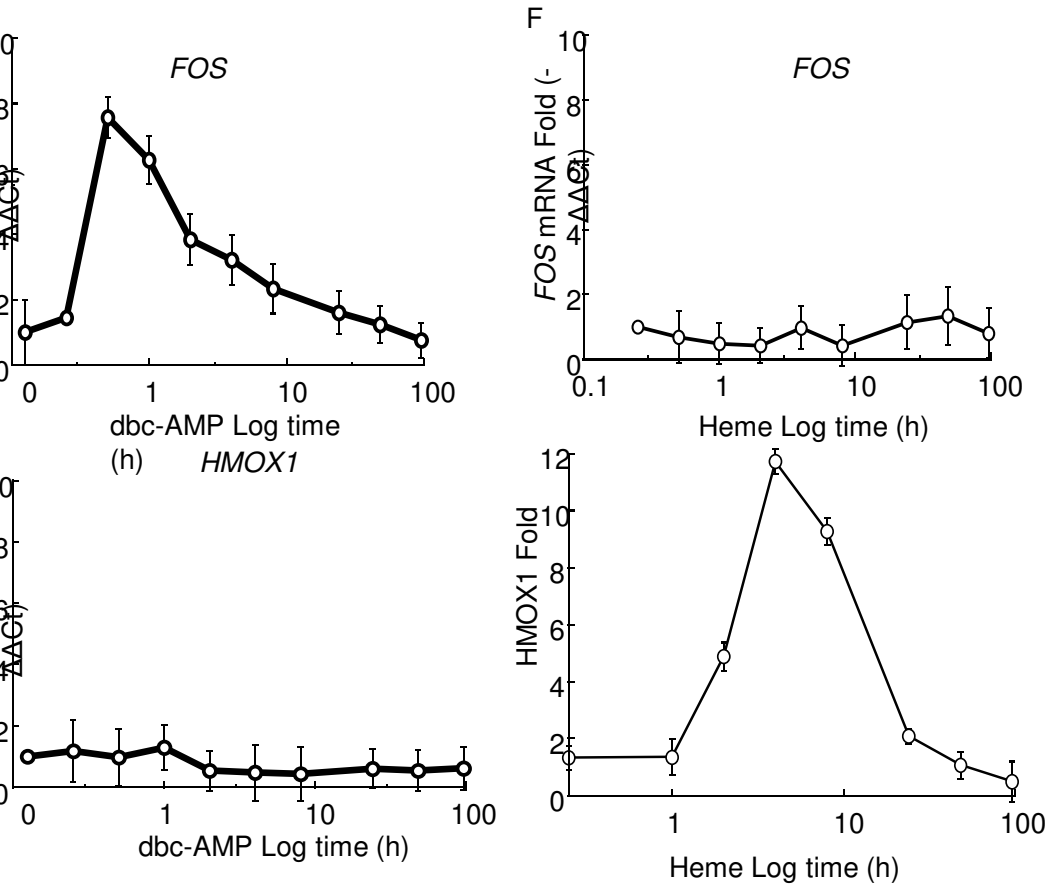

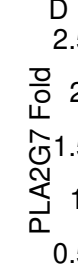
PLATGT
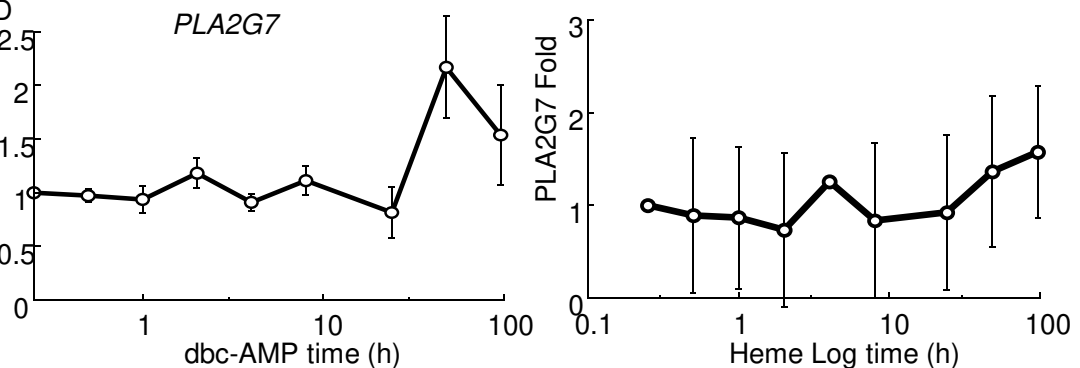

$\stackrel{1}{10} \stackrel{10}{H e m e}$ Log time $(h)$

Figure 2, Specificity of heme-induced genes and c-AMP-induced genes. Also, dbc-AMP measeres

Imperial College London

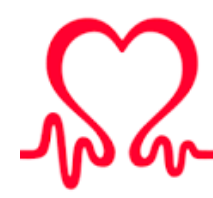

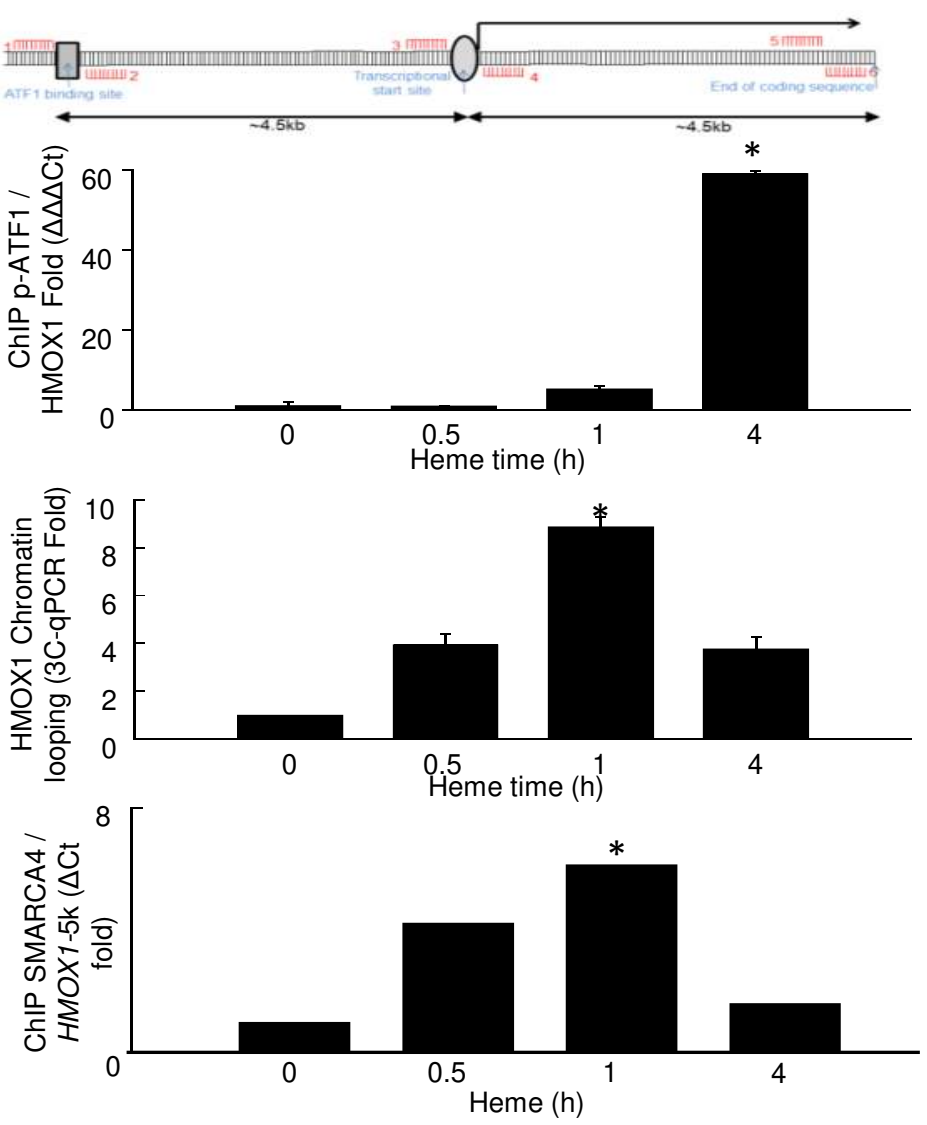
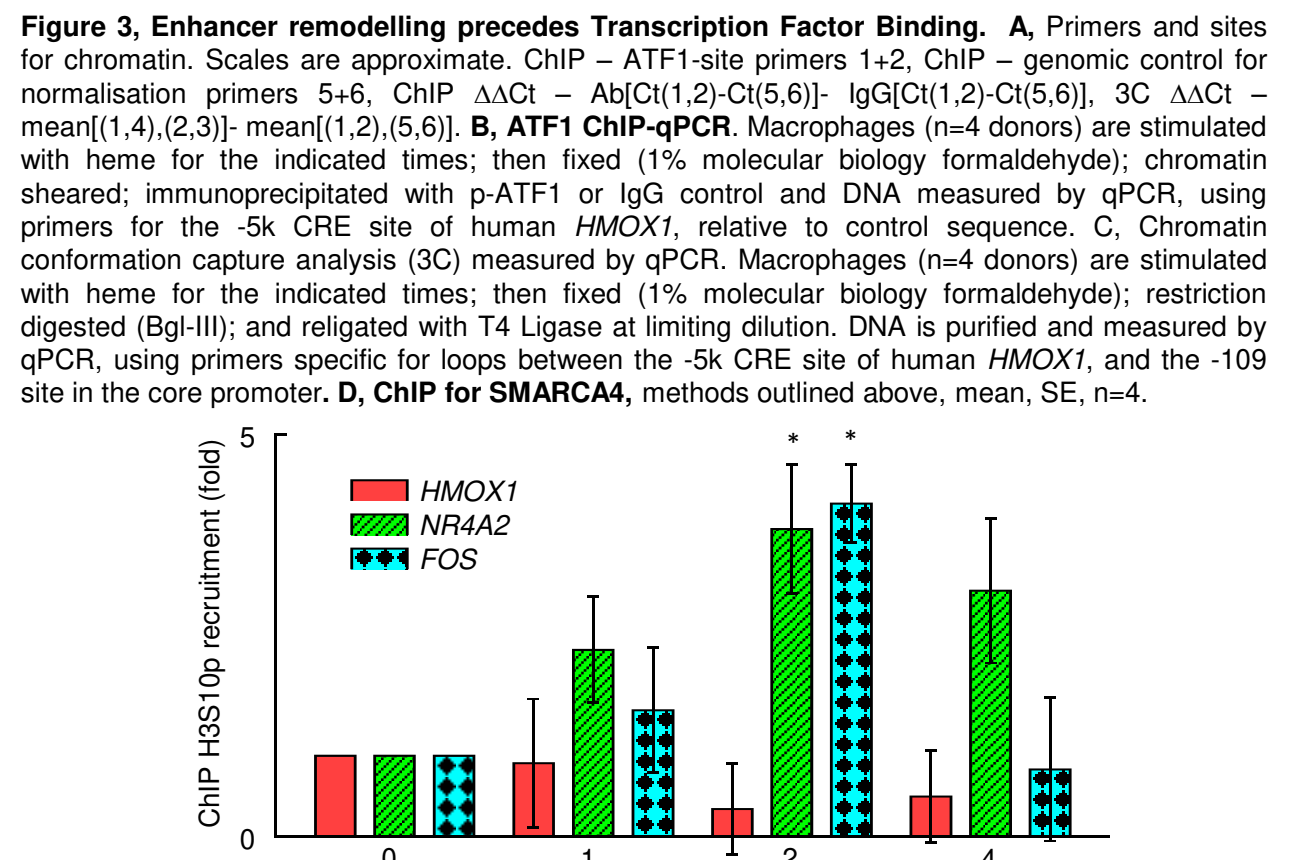

eme (h)

Figure 4, Chromatin Immunoprecipitation ChIP for phosphorylated Histone H3 instead, and

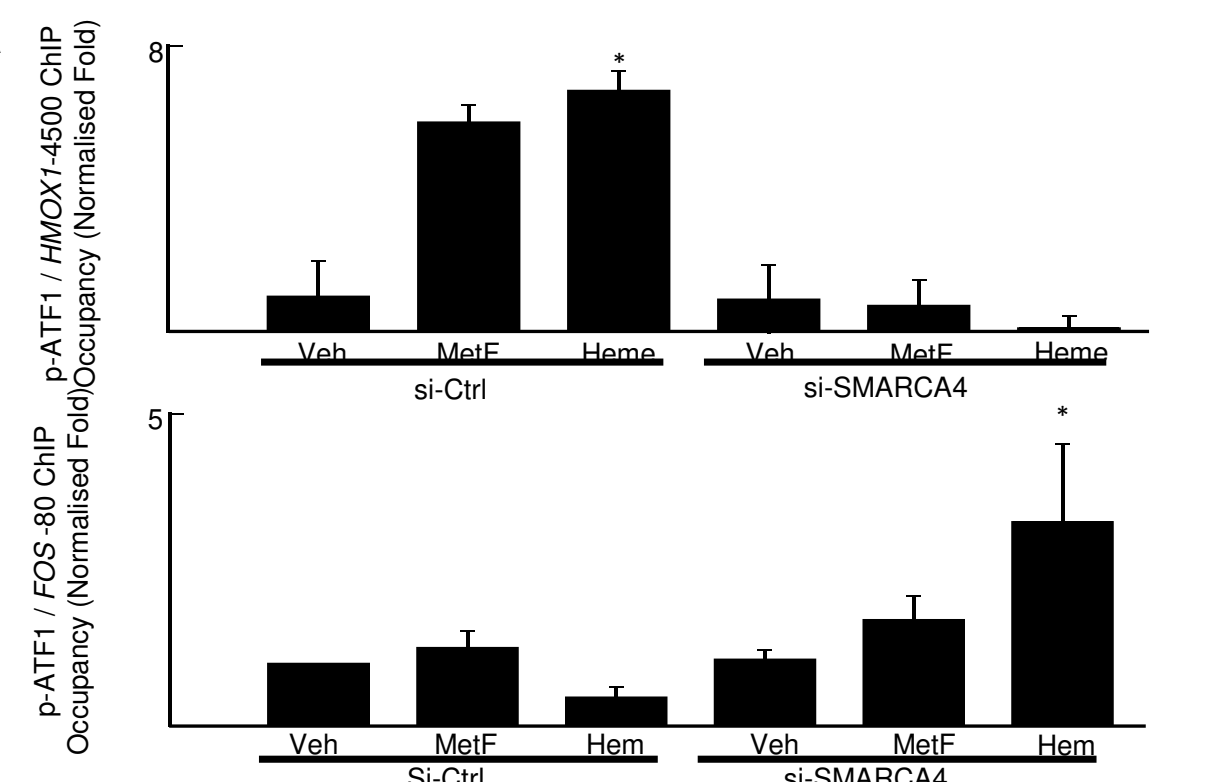

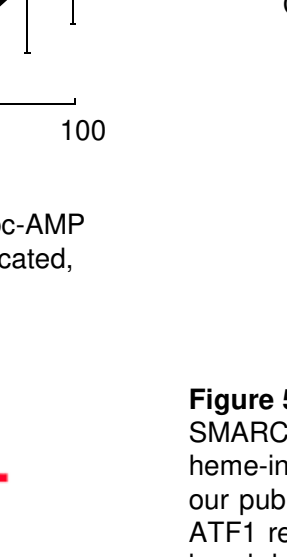
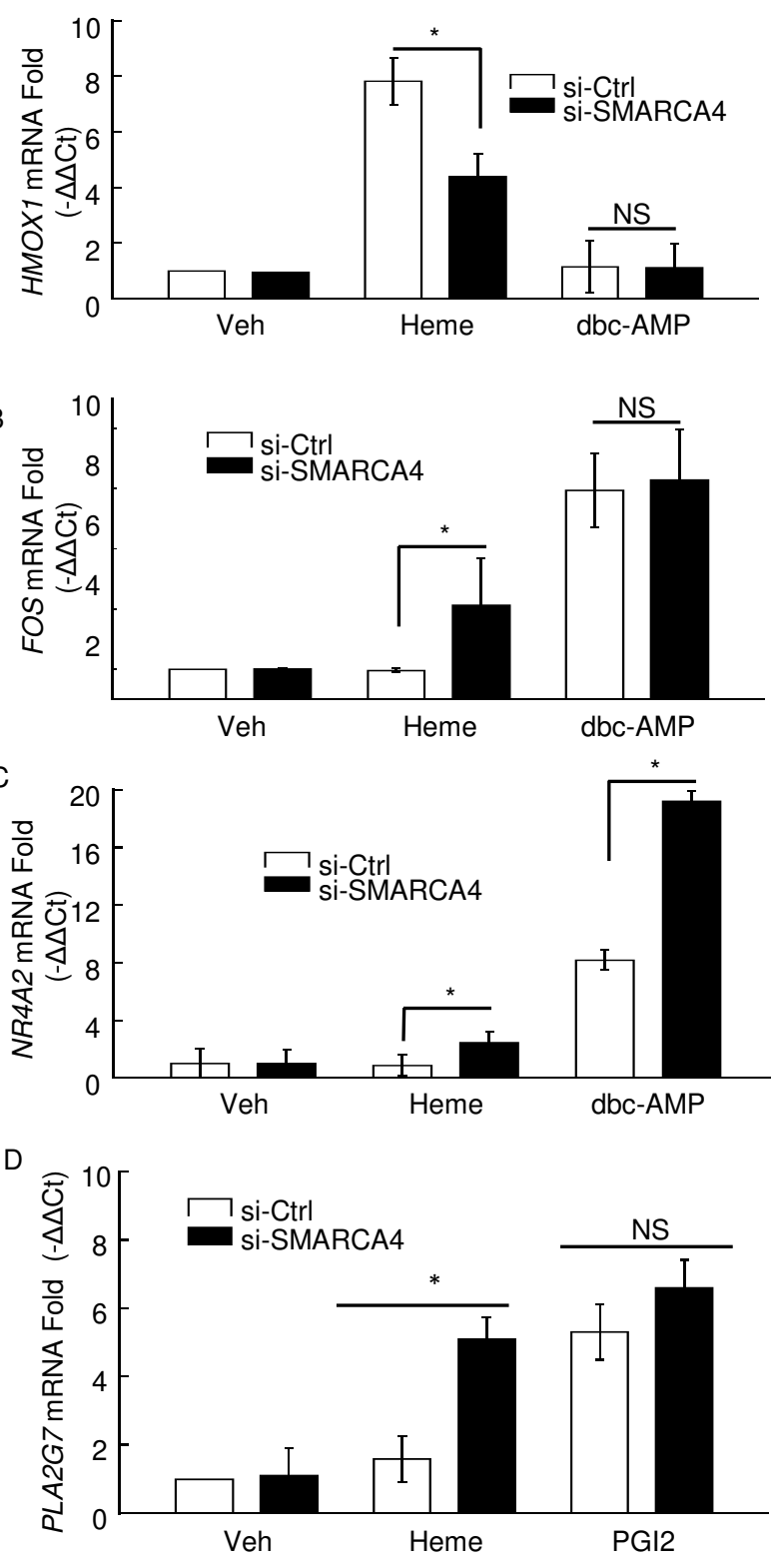

Figure 6 SMARCA4-knockdown rewires gene transcription producing hMDM ( $n=16$ donors), si-SMARCA4 reduces by si-SMARCA4 (*0<0 01 ). This indicates that Si-SMARCA4-induced redistribetion p-ATF1-produces functional differences in gene expression. Moreover,
SMARCA4-knockdown is does not generally suppress gene activation.

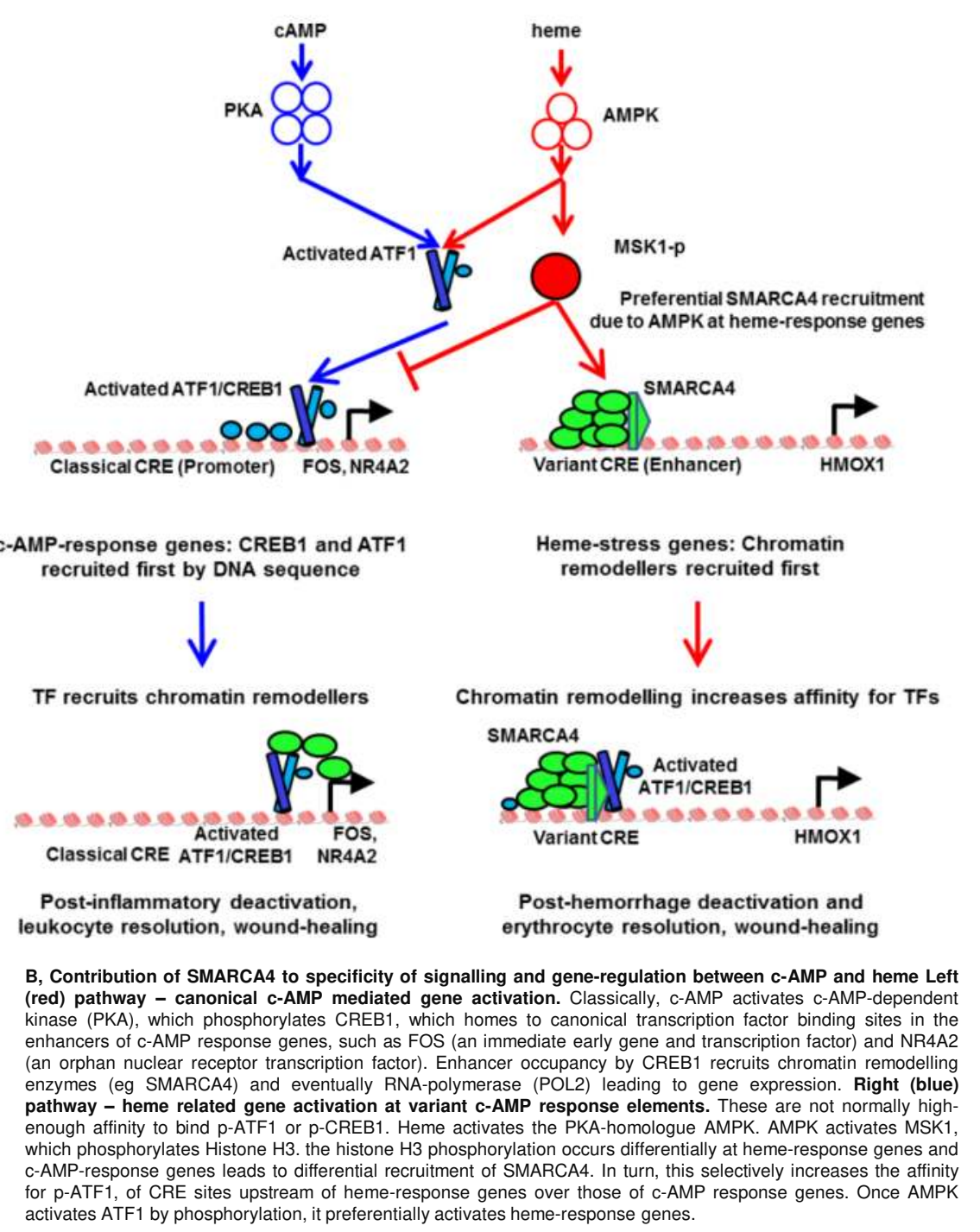

